\title{
Automated Guided Vehicles by Permanent Magnet Synchronous Motor: Future of In-house Logistics
}

Rao Anand ${ }^{1}$, Nihal Vishnu Vantagodi², Kartik A Shanbhag², Mahesh M ${ }^{1}$

${ }^{1}$ Department of Electrical Engineering, PES University, Bangalore, India

2Department of Electrical Engineering, PES Institute of Technology, Bangalore, India

Received January 10, 2019; Accepted February 5, 2019

Abstract: Material handling and logistics management that involve transportation of work pieces on production floor are important aspects to manufacturing that affect productivity and efficiency. Tow vehicles that are manually driven are currently used for this purpose. These processes can be better performed through automation. Automated guided vehicle (AGV) is an apt solution. AGVs are unmanned autonomous vehicles that can be programmed to perform versatile tasks. AGVs available in market are imported and hence have high capital cost and increased lead time for spare parts. Proposed AGV is built with a capital cost that is less than half of the existing AGVs. Its design is made indigenously, with most of its parts locally sourced. It can achieve a speed of $0.83 \mathrm{~m} / \mathrm{s}$, with a pulling capacity of $1,300 \mathrm{~kg}$. Its rechargeable batteries sustain four hours of continuous operation for one complete discharge. It has been tested and found to effectively replace tow vehicles.

Keywords: Automated guided vehicle • Power system • System engineering • Performance reliability • Component robustness

\section{Introduction}

\subsection{Significance}

The 21st century industrial revolution has been dominated by advances in robotics and automation. While once there were human labourers toiling on shop floors, they have now been replaced with advanced robots that perform the same tasks with increased efficiency and minimal human supervision. One such example is a common sight in large-scale manufacturing plants such as Tesla Giga factory, Boeing Fuselage Automated Upright Build Factory, and Toyota Motors. Not being limited to manufacturing sector, automated guided vehicles (AGVs) have also become inseparable part of warehouse management, pharmaceutical and healthcare, food and beverages, textiles, retail, and many more. With their market valued at USD 26 billion in 2015 and expected to reach USD 44 billion by 2022 (Jensfelt et al., 2001; Graf et al., 2004), AGVs will remain to be in the forefront of industrial revolution in years to come.

The AGVs, as defined by Material Handling Institute of America, are unmanned vehicles that move on wheels, carrying load on the plant floor, and are computer controlled and battery powered. AGVs, first introduced in 1953, were modified towing tractors used to pull trailers and follow overhead wires in grocery warehouses (Evans et al. 1992; Takahashi et al. 2010). AGVs today have come a long way and employ the latest guidance and control systems for their operation.

AGV serves as material handling and logistics management equipment that is used in the movement of work pieces between specified locations. In any manufacturing industry, factory logistics plays an important role affecting

\footnotetext{
*E-mail: anandprajna@gmail.com,nihalvantagodi@gmail.com, kartikshanbhag@gmail.com, matadamaheshu@gmail.com
} 
production efficiency and energy consumption. It involves movement of raw materials and finished goods/products between factory warehouse and the production shop floor: any bottleneck and inefficiency in factory logistics results in poor productivity of the whole factory. Unmanned AGVs have replaced human-driven forklifts and tow vehicles that were earlier used for this purpose. Various types of AGVs are tugging vehicle, unit load vehicle, automated fork lift, automated carts etc. (Vivaldini et al., 2010; Miller et al., 1994). The AGV proposed in this article is a tugger AGV that is specialized in towing multiple trailers (dolly) (Tasaki et al., 2015).

\subsection{Need for AGVs}

Need for AGVs is prevalent in all countries that strive to improve the efficiency of the manufacturing sector. In a developing country like India, there is an increase in emphasis on improving its manufacturing sector and this is supported by various government initiatives. AGVs have the potential to play a pivotal role in this context. The Make in India campaign aims at increasing indigenous manufacturing and production of commodities. To achieve this, Indian industries need to compete with the global market. However, India is behind the curve on deployment of automation technologies in manufacturing and maintenance. Less than $1 \%$ of India's manufacturing gross national product (GDP) comes from automation and instrumentation, whereas in developed economies it is above $5 \%$ (Frost and Sullivan, 2013). Bridging this gap is possible only with enhanced investment in automation, of which AGVs are an integral part. The Skill India campaign aims at developing and upgrading the skill levels of the working human capital. Currently in India, due to the lack of automation, a section of the work force is involved in low skilled, repetitive tasks, of which material handling is also a part, which is driving tow vehicles and fork lifts. These tasks do not improve skills and have the potential of being automated with the help of AGVs. By implementing AGVs for repetitive tasks, the work force can instead be better utilised in more challenging roles. The AGVs that have a gyroscope-based magnetic navigation system can achieve accuracy in positioning up to $\pm 5 \mathrm{~mm}$ (Ullrich, 2015). This accuracy level cannot be matched by humans. The AGVs can work 24/7 hours with uncompromised performance, making them best suitable for industrial applications.

\section{Proposed method}

The proposed AGV in this article is a "Tugger AGV". The specifications of the AGV are listed in Table 1.

Table 1. General specification of AGV

\begin{tabular}{ll}
\hline Description & Value \\
\hline \hline Speed & $3 \mathrm{~km} / \mathrm{h}$ \\
Maximum pulling capacity & $1,300 \mathrm{~kg}$ \\
Endurance & Four hours of continuous operations before a full discharge \\
Dimension (length $\times$ width $\times$ height) & $1,800 \mathrm{~mm} \times 380 \mathrm{~mm} \times 200 \mathrm{~mm}$ \\
\hline
\end{tabular}

AGV, automated guided vehicle.

The various blocks of AGV are shown in Figure 1 and the same are explained in detail in the following sections.

\subsection{Power supply unit}

It is used to power the various components of the AGV. It is composed of batteries, autocharging unit, DC-DC converter, and a circuit breaker (CB). The batteries used are lithium iron phosphate (LFP), a type of lithium ion batteries that are light weight and compact with high energy density. These are recharged by means of an autocharging unit that is placed in the AGV path. AGVs are programmed to stop at the charging station, wherever an opportunity to charge is available. To ensure a steady supply of voltage from the batteries, independent of state of charge of battery, DC-DC converter is used. To protect the various devices in the case of a fault or short circuit, $\mathrm{CB}$ is used as a protective element.

\subsection{Control unit}

Control and co-ordination of the AGV are achieved by means of control unit. The controller used is a programmable logic controller (PLC), programmed using ladder logic. It controls the differential speed mechanism of the drive 


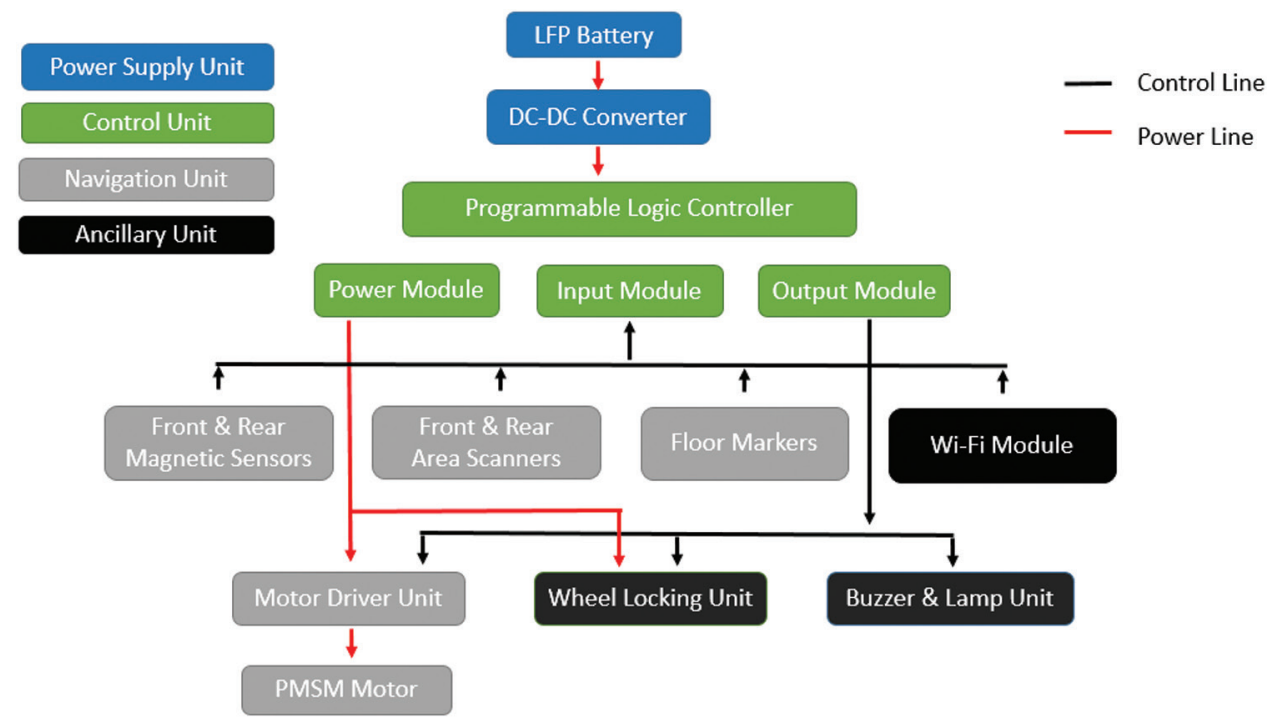

Fig. 1. Various blocks of automated guided vehicle.

unit by varying the speed of the drive wheels depending on the turning radius of the AGV path. It optimises the functioning of area sensors, which are used to sense the obstacles, by modifying their range in accordance with the path layout. The PLC counter programme keeps a count of the floor markers and triggers the working of the peripheral units as and when the markers are sensed.

\subsection{Navigation unit}

It enables the AGV to move along the desired direction while avoiding obstacles. It consists of the following.

Drive unit: It is composed of two permanent magnet synchronous motors (PMSMs) that individually drive two wheels. The steering of the drive unit is achieved by differential speed mechanism. In differential speed mechanism, one of the drive wheels needs to rotate at a relatively slower or faster RPM compared to the other drive wheel, for the AGV to turn.

Magnetic tape and guide path sensor: Magnetic tape is laid on the floor along which the AGV needs to travel. The guide path sensors are magnetic sensors that are used to detect the magnetic tape and work on the principle of Hall Effect. They are robust, durable, and hence suitable to be laid in industrial area (Anand et al., 2018; Pang et al., 2008; Fujimoto et al., 2001).

Floor markers: Various instructions to the AGV such as starting, stopping, loading, unloading, off-lining, charging etc. are given by means of the floor markers. Floor markers are strips of South Pole magnetic tapes that are laid at predefined locations along the path. The markers are sensed as counter inputs, which when sensed increase the programme count and execute a set of instructions that include but are not limited, such as speed of the vehicle, changing the range of the sensors etc.

Area sensors: AGVs work in an environment comprising other machines and humans. They are equipped with area sensors that are safety devices that are capable of detecting objects around them. Whenever an obstacle is sensed, the AGV either slows down or stops depending on the distance at which the obstacle is sensed.

\subsection{Ancillary units}

These units add to the functionality of the AGV and are specific to the proposed AGV that is used to pull the load. This unit enables the AGV to latch onto the dolly by means of a retractable connecting pin.

Wheel locking unit: AGVs also consist of four driven wheels; two each on front and rear sides. These must be either locked or free to swivel depending on the direction of movement. Wheel locking unit controls this, ensuring stability during bidirectional movement. It locks the rear wheels while moving in the forward direction and allows the front wheels to swivel according to the two motor-powered drive wheels. During backward movement the vice versa will happen, ensuring proper movement of AGV. 
Warning system: It consists of a tower and buzzer lamp that has different audio and visual signals, indicating the presence and proximity of obstacles. Three different colours are used as follows: green: when AGV is moving at intended speed; yellow: when AGV is moving at a slower speed because of an obstacle sensed at a sufficient distance; and red: when the AGV stops because of obstacle being very close to the AGV.

Wi-Fi unit: This unit is necessary when there are multiple AGVs working in the same path. Wireless communication is used to transmit and receive data between the AGVs and control panel. This is meant to serve two main purposes: 1) as a traffic management system that controls the movement of various AGVs and 2) as a means for peripheral units in the line to communicate with AGVs.

\section{Power solution for AGV}

The power unit is the most prominent system of the AGV as it involves a significant portion of the capital cost and is the only source of running cost in AGVs. The possible technologies for powering the AGV are lead acid batteries, LFP batteries, and ultra capacitor-battery hybrid system.

\subsection{Lead acid battery}

This is the conventional method used to power AGVs. Its main advantages are low cost and easy availability. However, it has several disadvantages such as low specific energy, lesser number of cycle life, slower charging time, and heavy weight. Hence, it is not suitable to be used in the proposed AGV.

\subsection{Ultracapacitor-battery hybrid system}

This hybrid power system is an emerging technology used to power AGVs. The uniqueness of this technology are increased efficiency and longevity. The high dynamic or transient current demand is taken by the ultra capacitor while the steady state load is taken by the battery (Pavkovic et al., 2014).

There are three topologies that are used in the battery-ultracapacitor hybrid system as follows: passive, semiactive, and fully active. Passive topology involves direct coupling of load with the Ultra capacitor bank and the battery in parallel (Dougal et al., 2002). This system is trade-off for performance with cost. Semiactive topology enhances the performance of passive topology at the cost of additional control circuitry and DC-DC converter (Ortuzar et al., 2007). The ultimate performance is achieved with complex circuitry involving two DC-DC converters in fully active topology. But this is a trade-off with cost, simplicity, and weight (Camara et al., 2010).

\subsection{Lithium battery}

Dubbed as the most suitable power solution system for electric vehicles, the LFP battery comes with a large number of advantages such as high energy density, long lifespan, and more number of cycles with greater depth of discharge. They are very safe even while charging/discharging at higher rate (Patel et al., 2015). They have low self-discharge and require minimal maintenance.

\subsection{Comparisons}

In Figure 2, a comparison of different technologies based on the given technical parameters is made. The hybrid system has high specific power, high efficiency, and more cycle life compared to other systems. On the other hand, LFP batteries have higher specific energy (around 10 times that of hybrid system and 4 times that of lead acid batteries). Even at higher depth of discharge, the LFP batteries can be used. The charging time varies in the order of few minutes for the hybrid system to a few hours for lead acid and LFP batteries.

Comparison of different technologies based on the general parameters is shown in Figure 3 . Hybrid system and LFP batteries are very safe to operate and have wide operating temperature range. On the other hand, lead acid batteries are unsafe due to the risk of explosion when overcharged. Lead acid batteries weigh more and have least Watts to weight ratio of all three systems. Hybrid systems are seldom available for commercial purposes in developing countries, as they are an emerging technology while LFP and lead acid batteries are easily accessible.

Figure 4 is a cost comparison analysis which shows that hybrid systems have very high initial cost (around 10 times of LFP batteries) and highest energy cost (cost $/ \mathrm{kW})$, but lesser maintenance cost. The lead acid battery being cost effective but has several drawbacks. From the comparison, we can conclude that LFP batteries are the most suitable solution for the AGVs. 


\section{Energy Storage technologies}

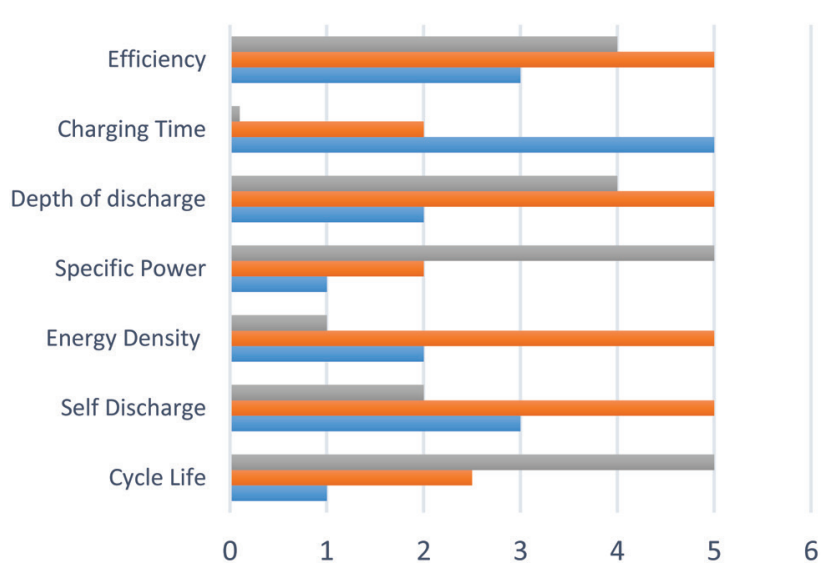

Ultracap-Battery Hybrid $\quad$ LFP Battery $\quad$ Lead Acid

Fig. 2. Comparison of energy storage technologies.

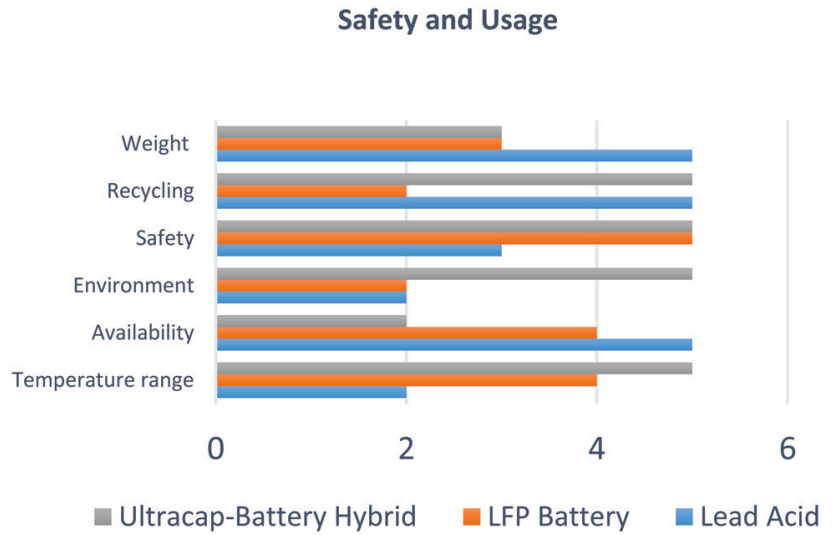

Fig. 3. Comparison of safety and usage.

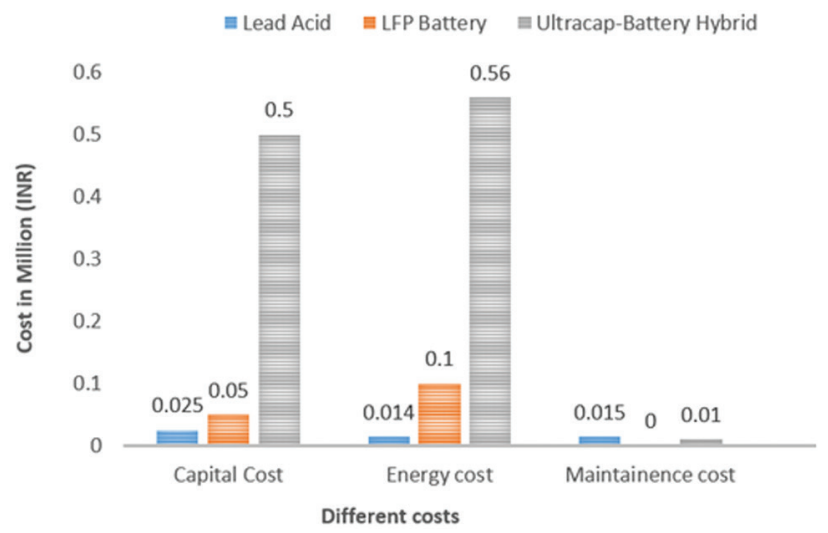

Fig. 4. Cost comparison of various power solutions. 


\section{Advantages of AGV}

AGVs offer inherent flexibility and scalability. They can be easily integrated with new or existing material handling equipment to develop a fully automated system. As operations expand, new AGVs can be added without significant changes to the existing system. AGVs reduce labour costs as they redistribute work force to value added positions. They can work round the clock without the need for breaks or vacation time. They have little down time and operate at fixed rate to maintain efficient operational activity. Material handling involves operation in conditions that may not be suitable for human operations such as extreme temperature and hazardous environments. AGVs act as substitutes in these scenarios and keep staff out of danger. Large initial monetary investments are involved in the purchase of AGVs. However, as output production is increased and time and labour costs reduced, the efficiency of using AGVs is well worth the investment.

\section{Measurement and experimental validation}

\section{Experiment on Proto}

The model equations of the PMSM have been developed with rotor reference frame assuming that eddy current and hysteresis losses are negligible, hence the voltage equations are given by:

$$
\begin{aligned}
& V_{d}=R i_{d}-\lambda_{q} \omega_{r}+\rho \lambda_{d} \\
& V_{q}=R i_{q}+\lambda_{d} \omega_{r}+\rho \lambda_{q}
\end{aligned}
$$

The flux linkages of direct and quadrature axes are as follows:

$$
\begin{aligned}
& \lambda_{q}=L_{q} i_{q} \\
& \lambda_{d}=L_{d} i_{d}+\lambda_{f}
\end{aligned}
$$

Combining voltage and flux in matrix form is represented as follows:

$$
\left[\begin{array}{c}
V_{d} \\
V_{q}
\end{array}\right]=\left[\begin{array}{cc}
-\omega_{r} L_{q} & R+\rho L_{d} \\
R+\rho L_{q} & \omega_{r} L_{d}
\end{array}\right]\left[\begin{array}{l}
i_{d} \\
i_{q}
\end{array}\right]+\left[\begin{array}{c}
\rho \lambda_{f} \\
\lambda_{f} \omega_{r}
\end{array}\right]
$$

The torque developed by the motor is given by:

$$
\begin{aligned}
& T_{e}=1.5 P\left(\lambda_{d} i_{q}+\lambda_{q} i_{d}\right) \\
& T_{e}=T_{L}+B \omega_{m}+J \frac{d}{d t} \omega_{r}
\end{aligned}
$$

where, $R$ is the stator resistance in $\Omega ; i_{d}$ and $i_{q}$ are the $d$-axis and $q$-axis currents in $\mathrm{A} ; \lambda_{d}, \lambda_{f}$, and $\lambda_{q}$ are the flux linkages of $d$-axis, field, and $q$-axis due to the permanent magnets in $\mathrm{Wb} ; \omega_{r}$ is the electrical speed in rad/s; $J$ is the moment of inertia in $\mathrm{kg} \mathrm{m} \mathrm{m}^{2} ; T_{e}$ is the torque developed by the motor in $\mathrm{Nm} ; L_{q}$ and $L_{d}$ are $q$-axis and $d$-axis inductances in $\mathrm{H} ; B$ is the mechanical friction in $\mathrm{N} ; \omega_{m}$ is the rotor speed in rad/s; $T_{L}$ is the load torque in $\mathrm{Nm}$; and $\rho$ is the derivative operator.

At any time $t$, the rotating rotor $d$-axis makes an angle $\theta$ with the fixed stator phase axis and rotating stator magneto motive force (MMF) makes an angle $\alpha$ with the rotor $d$-axis, stator MMF rotates at the same speed as that of rotor (Wang et al., 2014; Cao et al., 2012). 
Table 2 represents the selected sample for hardware development, and output has been represented in Figure 5. Figure 6 represents the plots of torque, jerk output with vibrations of the sample.

Table 2. Sample motor parameters

\begin{tabular}{lc}
\hline Particulars & Sample \\
\hline \hline Speed & $3 \mathrm{~km} / \mathrm{h}$ \\
Maximum pulling capacity & $1,300 \mathrm{~kg}$ \\
\hline
\end{tabular}

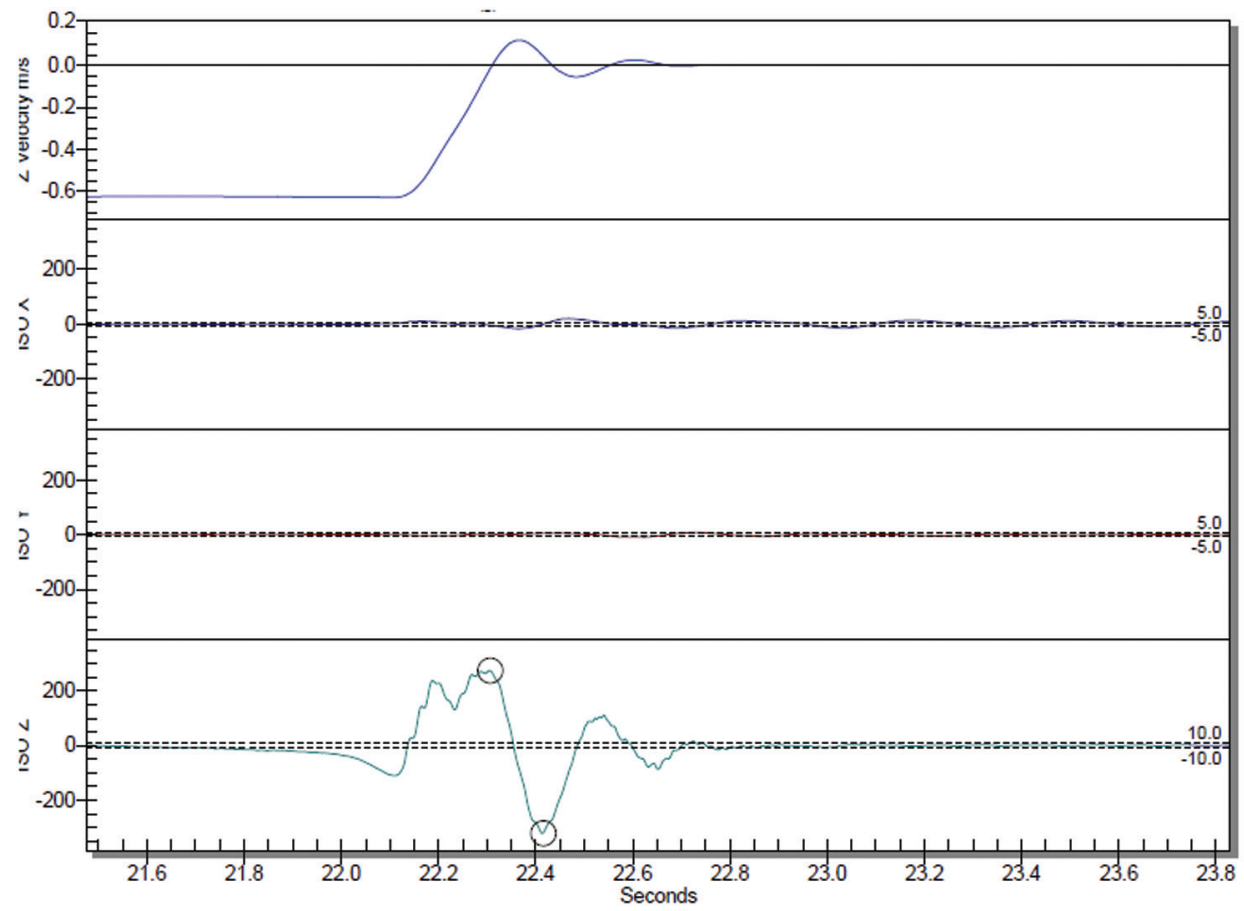

Fig. 5. Torque profiles with vibrations.

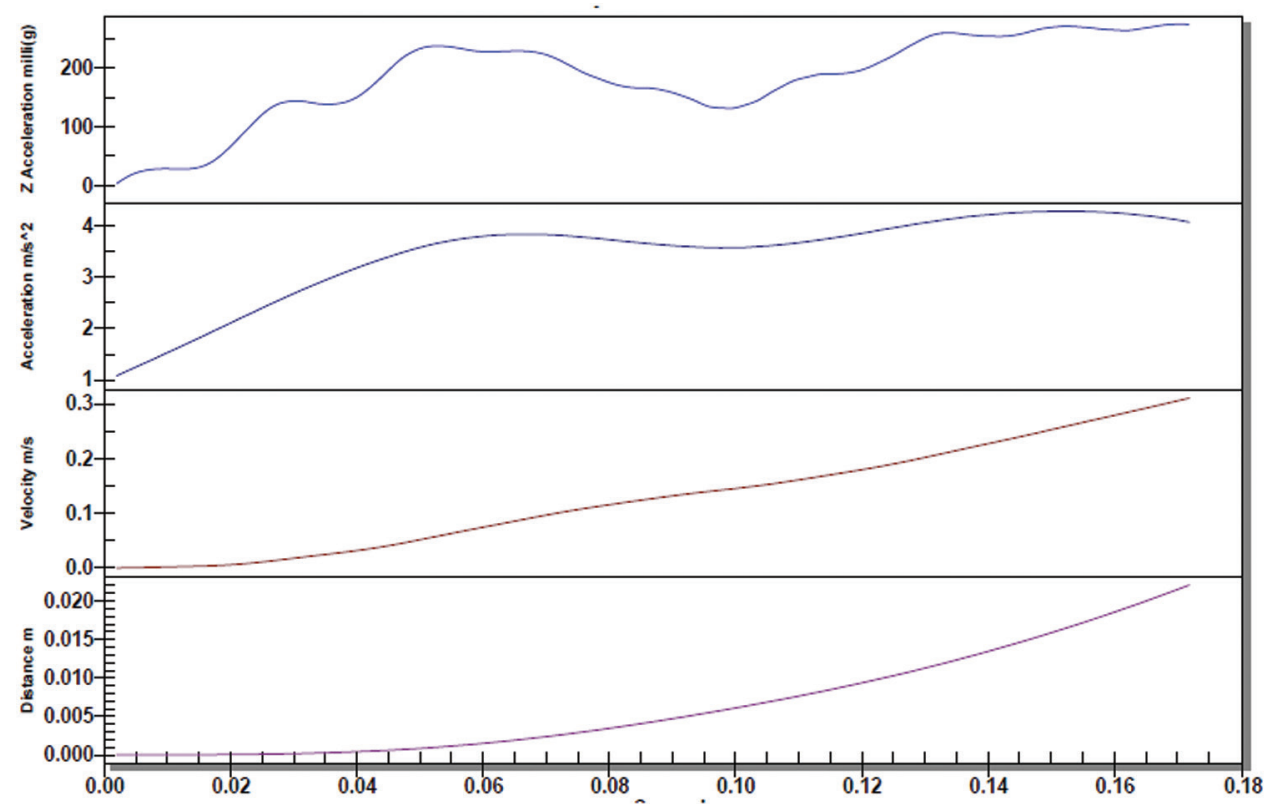

Fig. 6. Jerk and vibration analysis. 


\section{Conclusion}

AGVs are an effective substitute to any industry that employs the use of material handling equipment. Given the repetitive functionality of these equipment, there is immense scope for automating them by PMSM. The classical speed controlling technique of the PMSM machine can be improved with modern techniques, such as controller using fuzzy logic and neural networks. Automation through AGVs is a flexible, versatile solution that will not only result in elimination of human labour costs but also make the process more safe, efficient, and accurate. Research and development in the area of AGV navigation and control are ongoing. It has resulted in the development of cutting edge, advanced technologies such as laser guidance, camera-based imaging, Simultaneous Localization and Mapping (SLAM algorithm) (Koch et al., 2017; McDonald et al., 2013; Grisetti et al., 2010), GPS/Dead Reckoning (DR) system, Fuzzy inference system and Neural Network Technique for navigation, and many more. These advancements will help in further development of AGV driven by PMSM technology and make them more reliable and efficient.

\section{References}

Camara, M. B., Gualous, H., Gustin, F, Berthon, A. and Dakyo, B. (2010). DC/DC Converter Design for Super capacitor and Battery Power Management in Hybrid Vehicle Applications Poly- nomial Control Strategy. IEEE Transactions on Industrial Electronics, 57(2), pp. 587-597.

Cao, W. P., Mecrow, B. C., Atkinson, G. J., Bennnett, J. W. and Atkinson, D. J. (2012). Overview of Electric Motor Technologies Used for More Aircraft (MEA). IEEE Transactions on Industrial Electronics, 59(2), pp. 803-811.

Dougal, R. A., Liu, S. and White, R.E. (2002). Power and Life Extension of Battery-Ultracapacitor Hybrids. IEEE Transactions on Components and Packaging Technologies, 25(1), pp. 120-131.

Evans, J., Krishnamurthy, B., Barrows, B., Skewis, T. and Lumelsky, V. (1992). 'Handling Real-World Motion Planning: A Hospital Transport Robot. IEEE Control Systems, 12(1), pp. 15-19.

Frost and Sullivan (2013) Automation Market to Reach USD 2000 Million by 2016 - Frost and Sullivan, CEW India Publication, Feb 2013

Fujimoto, T., Ota, J, Arai, T., Ueyama, T. and Nishiyama, T. (2001). Semi-guided navigation of AGV through iterative learning. In Intelligent Robots and Systems, 2001. Proceedings. 2001 IEEE/RSJ International Conference on, volume 2, pp. 968-973. IEEE.

Graf, B., Hans, M. and Schraft, R. D. (2004). Care-Obot II-Development of a Next Generation Robotic Home Assistant. Autonomous Robots, 16(2), pp. 193-205.

Grisetti, G., Kümmerle, R., Stachniss, C. and Burgard, W. (2010). A tutorial on graph-based SLAM. The IEEE Intelligent Transportation Systems Magazine, 2(4), pp. 31-43.
Jensfelt, P. and Kristensen, S. (2001). Active Global Localization for a Mobile Robot Using Multiple Hypothesis Tracking. IEEE Transactions on Robotics and Automation, 17(5), pp. 748-760.

Koch, R., May, S., Murmann, P. and Nüchter, A. (2017). Identification of transparent and specular reflective material in laser scans to discriminate affected measurements for faultless robotic SLAM. Robotics and Autonomous Systems, 87, pp. 296-312.

McDonald, J., Kaess, M., Cadena, C., Neira, J. and Leonard, J. J. (2013). Real-Time 6-DOF Multi-Session Visual SLAM Over Large-Scale Environments. Robotics and Autonomous Systems, 61(10), pp. 1144-1158.

Miller, R. K., Stewart, D. G., Brockman, W. H. and Skaar, S. B. (1994). A Camera Space Control System for an Automated Forklift. IEEE Transactions on Robotics and Automation, 10(5), pp. 710-716, doi: 10.1109/70.326575

Ortuzar, M, Moreno, J. and Dixon, J. (2007). Ultracapacitor-Based Auxiliary Energy System for an Electric Vehicle: Implementation and Evaluation, IEEE Transactions on Industrial Electronics, 54(4), pp. 2147-2156.

Pang, Y., De La Cruz, A. L. and Lodewijks, G. (2008). Bipolar magnetic positioning system for automated guided vehicles. Intelligent Vehicles Symposium, 2008 IEEE, pp. 883-888. IEEE.

Patel, D. D. and Salameh, Z. M. Characterization of GP30EVLF 30 Ah Lithium Iron Phosphate Battery Cells. Power \& Energy Society General Meeting, 2015 IEEE, pages 1-5. IEEE, 2015.

Pavkovic', D., Lobrovic', M., Hrgetic', M. and Komljenovic', A. (2014) A Design of DC Bus Control 
System for EVs Based on Battery/Ultracapacitor Hybrid Energy Storage. In Electric Vehicle Conference (IEVC), 2014 IEEE International, pages 1-8. IEEE.

Raghavendra Rao, A., \& Mahesh, M. (2018). Analysis of the energy and safety critical traction parameters for elevators. EPE Journal, 28(4), 169-181.

Takahashi, M., Suzuki, T., Shitamoto, H., Moriguchi, T. and Yoshida, K. (2010). Developing a Mobile Robot for Transport Applications in the Hospital Domain, Robotics and Autonomous Systems, 58(7), pp. 889-899.

Tasaki, R., Kitazaki, M., Miura, J. and Terashima, K. (2015). Prototype Design of Medical Round Supporting Robot 'Terapio,' in Proceedings of IEEE IEEE International Conference on Robotics and Automation (ICRA), Seattle, WA, USA, pp. 829-834.

Ullrich, G. (2015). Automated Guided Vehicle Systems: A Primer with Practical Applications. Berlin: Springer, pp. 38-40, 2nd revised edition

Vivaldini, K. C. T. et al. (2010). Robotic forklifts for intelligent warehouses: Routing, path planning, and auto-localization. 2010 IEEE International Conference on Industrial Technology, Vina del Mar, pp.1463-1468. doi: 10.1109/ICIT.2010.

Wang, K., Zhu, Z. Q., Grzegorz Ombach, Koch, M., Zhang, S. and Xu, J. (2014). Electromagnetic Performance of an 18-Slot/10-Pole Fractional-Slot Surface-Mounted Permanent-Magnet Machine. IEEE Transactions on Industry Applications, 50(6), pp. 3685-3696. 